\title{
Lab-on-a-Chip Devices for Point-of-Care Medical Diagnostics
}

\author{
Sofia Arshavsky-Graham and Ester Segal
}

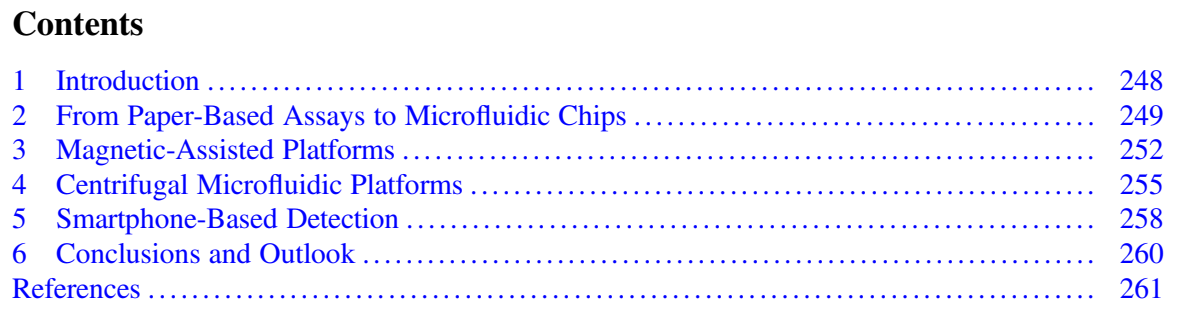

\begin{abstract}
The recent coronavirus (COVID-19) pandemic has underscored the need to move from traditional lab-centralized diagnostics to point-of-care (PoC) settings. Lab-on-a-chip (LoC) platforms facilitate the translation to PoC settings via the miniaturization, portability, integration, and automation of multiple assay functions onto a single chip. For this purpose, paper-based assays and microfluidic platforms are currently being extensively studied, and much focus is being directed towards simplifying their design while simultaneously improving multiplexing and automation capabilities. Signal amplification strategies are being applied to improve the performance of assays with respect to both sensitivity and selectivity, while smartphones are being integrated to expand the analytical power of the technology
\end{abstract}

\footnotetext{
S. Arshavsky-Graham

Department of Biotechnology and Food Engineering, Technion - Israel Institute of Technology, Haifa, Israel

Institute of Technical Chemistry, Leibniz University Hannover, Hanover, Germany

E. Segal $(\triangle)$

Department of Biotechnology and Food Engineering, Technion - Israel Institute of Technology, Haifa, Israel

The Russell Berrie Nanotechnology Institute, Technion - Israel Institute of Technology, Haifa, Israel

e-mail: esegal@technion.ac.il
} 
and promote its accessibility. In this chapter, we review the main technologies in the field of LoC platforms for PoC medical diagnostics and survey recent approaches for improving these assays.

\section{Graphical Abstract}

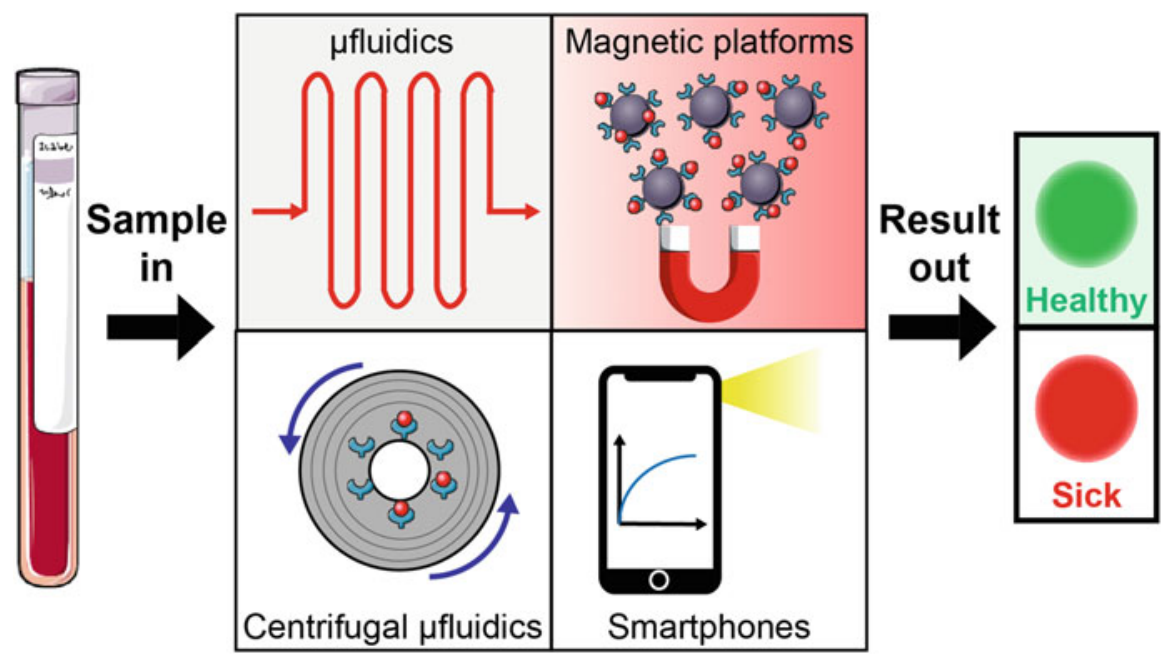

Keywords Centrifugal microfluidics, Diagnostics, Lab-on-a-chip, Microfluidics, Paper, Point-of-care, Smartphone

\section{Introduction}

Over the years, medical diagnostics has been shifting away from imaging and invasive tissue sampling, towards far less invasive tests that detect disease biomarkers in extracted body fluids. Such biomarkers may include small metabolites, nucleic acids, proteins, and cells [1, 2]. Today, most assays for biomarker detection are mainly performed at centralized labs - requiring trained personnel for operation of complex benchtop analyzers, with a correspondingly long time-to-result period. The latter consideration is critical with respect to many medical conditions, for which time is frequently of the essence [3]. In addition, at low resource environments, such analyzers are necessarily limited due to their high costs and the need for skilled operators. As a result, significant efforts are now being directed towards development of point-of-care (PoC) tests, which can be operated at the patient site by non-trained personnel [1, 4-7]. Such tests should provide accurate, sensitive, and specific results in a rapid manner (with an optimal time-to-result in the range of few seconds to few hours) at relatively low-cost. The ideal vision for such a test would be 
an independent and self-sustainable operation that allows a non-trained operator to load a sample of extracted body fluid (e.g., blood, urine, saliva, sweat, etc.) into the instrument and obtain informative results with minimal user intervention (i.e., sample in, result out). Fully integrated lab-on-a-chip (LoC) technologies, which incorporate all related analysis steps (including sample loading and preparation) in a single device, stand to significantly advance PoC medical diagnostics [1, 5, 8-13].

In this chapter, we provide an overview of the primary technologies in the field of PoC medical diagnostics. These include paper-based assays and microfluidics, magnetic-assisted detection, centrifugal microfluidics, and smartphone-based detection. We will highlight the main concepts and directions in each technology, provide several relevant examples from the past 3 years, discuss the main challenges in the field, and conclude by offering a future-oriented perspective.

\section{From Paper-Based Assays to Microfluidic Chips}

Lateral flow assays are widely used for PoC diagnostics. In these assays, a liquid sample containing the target analyte moves (via capillary forces) through various zones of polymeric strips, on which capture probes that can interact with the analyte are immobilized (see Fig. 1) [14, 15]. One of the most common lateral flow assays is the commercial pregnancy test for detecting human chorionic gonadotropin in urine - in which a sandwich-based immunoassay is performed, and detection of the target protein is realized via a color change, which can be observed with the naked eye [16-19]. The main advantages of lateral flow assays are their simplicity, ease of use, extended shelf life, and low-cost. However, lateral flow assays require numerous reagents and relatively large volumes of sample, and both multiplexing and the control of the flow rate pose challenges [16-19].

Microfluidic technology has been applied to address these limitations by enabling precise control of the flow by different microchannel geometries [19, 20]. Capillarydriven microfluidic chips have been used for PoC diagnostics of various analytes

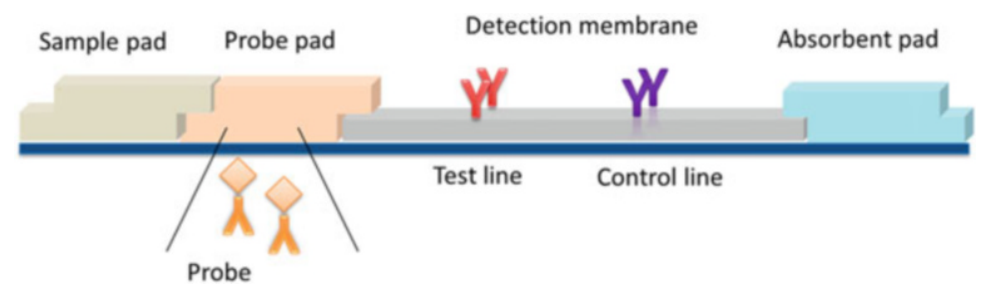

Fig. 1 Schematic illustration of a typical lateral flow assay strip. Few microliters of the sample are loaded to the sample pad and drawn to the probe pad, via capillary forces. The target is bound by labeled detection probes and transferred to the detection membrane and captured on a line of immobilized capture probes. Reprinted from Ref. [15] (Anfossi L. et al. Multiplex Lateral Flow Immunoassay: An Overview of Strategies towards High-throughput Point-of-Need Testing. Biosensors. 2018;9(1):2) 
[17, 21-24]. For example, the commercially available Triage system - which is comprised of a portable analyzing instrument and a disposable protein chip - aims to diagnose a wide variety of health conditions [25, 26]. Like the lateral flow immunoassay, a biological sample is loaded onto this chip, and the target antigen is first bound to labeled antibodies. The bound conjugates then pass through the detection zone, where they are captured by pre-immobilized antibodies. The cartridge is fabricated from polymer microfluidic channels, which result in lower batch-tobatch variability when compared to traditional lateral flow immunoassays. The capillary flow is passively controlled by the microstructure and surface characteristics, which increase the incubation time of the target with the detection zone in a controllable manner without the need for active pumps and valves. Thus, for a relatively low-cost, a simple and rapid ( $\sim 15 \mathrm{~min})$ detection platform is realized. Multiplexed biomarker detection from whole blood was achieved by the Lateral Flow Integrated Blood Barcode Chip [27]. This microfluidic chip, fabricated from a hydrophilic polymer bonded to a glass slide, includes an array of immobilized antibodies that are specific for a variety of protein biomarkers. A few microliters of whole blood with an anticoagulant are loaded onto the chip, and a filter paper is then used to draw the sample and other loaded reagents through the chip via action of capillary forces. Separation of blood cells from the plasma is achieved by inertial force. A wash buffer is used to reduce background noise by removing an unbound label. Each step in the assay is automatically and sequentially executed, and the whole assay is performed within the span of just $40 \mathrm{~min}$. To further automate the system, a self-coalescence module can also be integrated in a microfluidic chip, for the controlled reconstitution and delivery of inkjet-spotted and dried reagents. Welldefined reagent concentration profiles are established based on their initial spotting pattern [28]. This was applied in a silicon-based microfluidic chip for detection of a cardiac biomarker (troponin I) in human serum via a sandwich fluorescence immunoassay [16]. Figure 2a illustrates the platform, where a loading pad receives a sample, which is drawn by capillary forces to a self-coalescence module. The latter contains dried detection antibodies, which are reconstituted by a defined volume of the sample. That mixture then passes to a bead lane with capture antibodies, which selectively bind with the target-detection antibody complexes from the sample. The flow of the sample in the chip is controlled by a capillary pump. The design and image of the silicon microfluidic chip itself are presented in Fig. 2b, c, respectively. The assay requires $1 \mu \mathrm{L}$ of sample, performed within $25 \mathrm{~min}$, and a limit of detection of $4 \mathrm{ng} \mathrm{mL} \mathrm{m}^{-1}$ is realized.

An additional strategy for achieving reagent storage in paper-based microfluidic assays is seen in the use of a three-dimensional (3D) folding of a paper substrate with an origami-based technique. Different layers and dried reagents can be stacked vertically, and the addition of a buffer solution results in reconstitution via a controlled, multistep process. Parallel tests can be performed using a multilayer fluidic network in a compact device [18, 29]. Recently, 3D-origami-based paper device was used for detection of a biomarker for Staphylococcus aureus infection within human synovial fluid by an ELISA-based immunoassay [30]. That platform consists of a sliding strip and antibody storage functions on a single sheet of paper, as 

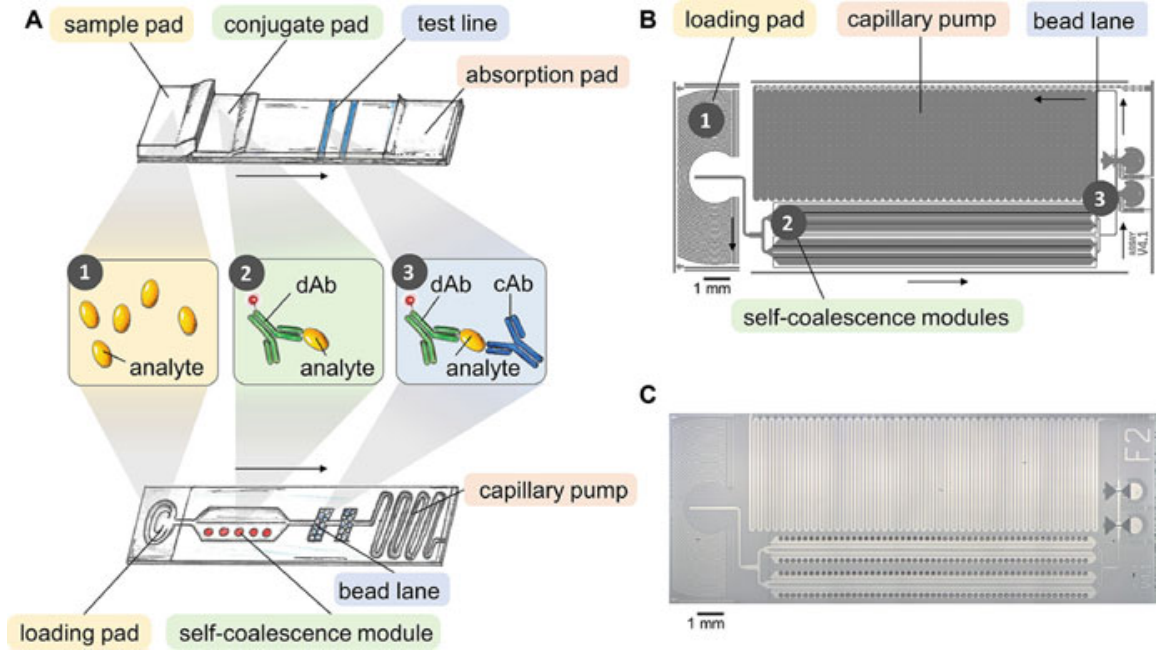

Fig. 2 (a) General concept of a lateral flow assay-based microfluidic chip, which integrates a selfcoalescence module, containing dried inkjet-spotted detection antibodies. The flow of the sample is driven by a capillary pump. (b) The design of the corresponding microfluidic chip and (c) an optical microscopy image of the fabricated Si microfluidic chip. Reprinted with permission from Ref. [16] (Hemmig E. et al. Transposing Lateral Flow Immunoassays to Capillary-Driven Microfluidics Using Self-Coalescence Modules and Capillary-Assembled Receptor Carriers. Analytical Chemistry. 2020;92(1):940-6). Copyright (2020) American Chemical Society

shown in Fig. 3a. The sliding strip acts as a valve to control the serial steps of sample addition, interaction, washing, and detection. The sequential flow is carried out by sliding the tab to different positions (see Fig. 3b). Only $3 \mu \mathrm{L}$ of sample are required, and this procedure can be completed within $7 \mathrm{~min}$. Nevertheless, the manual addition of buffers is still required during this procedure.

Sensitivity enhancement of lateral flow assays has frequently been achieved by incorporating various nanomaterials - such as gold or silver nanoparticles, magnetic nanoparticles, and quantum dots - into the system [19, 31, 32]. An alternate strategy is the use of external fields (i.e., acoustic, thermal, electric, etc.). Electrophoretic methods, such as ion concentration polarization or isotachophoresis, have also been applied to facilitate separation and concentration within microfluidic devices. In isotachophoresis, ionic species can be focused, based on their electrophoretic mobility, using a discontinues buffer system. The method enables the simultaneous extraction, separation, and concentration of the target species [33]. This method was recently applied for multiplexed detection of two cardiac biomarkers in human serum [34]. The platform is comprised of a lateral flow paper assembled on a 3D-printed cartridge for buffer reservoirs and electrode connection. The two protein targets are fluorescently labeled and detected by immobilized antibodies on the paper strip. The assay time is $6 \mathrm{~min}$ and results in $\sim 1,300$-fold enrichment of the proteins. Label-free detection with isotachophoresis in a microfluidic assay was demonstrated 
A
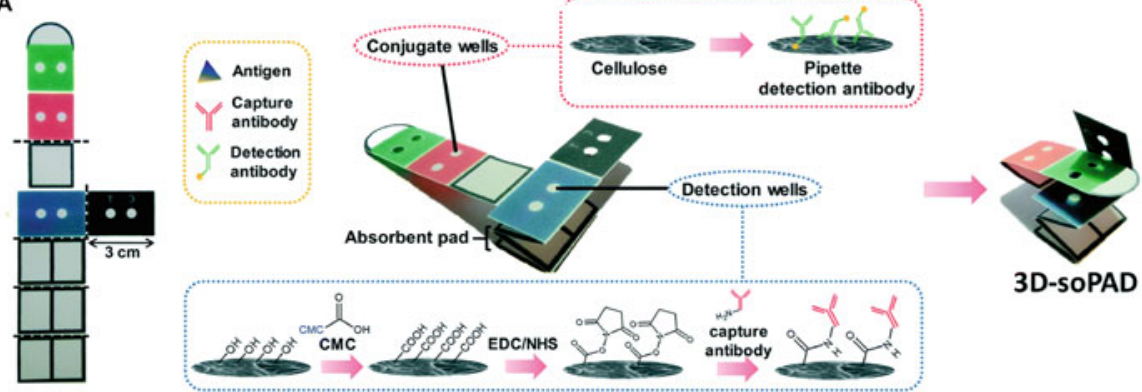

B

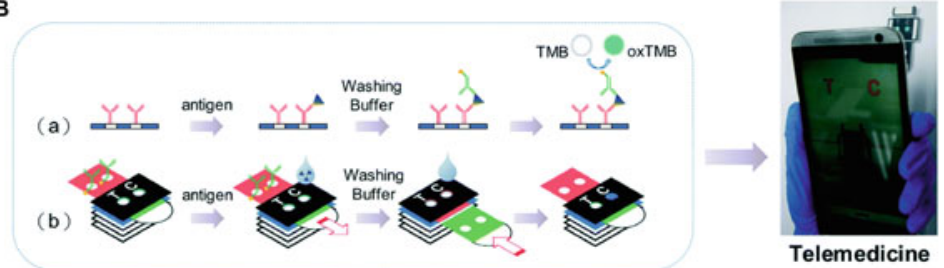

Fig. 3 3D-origami-based paper device used for detection of a protein A in human synovial fluid: (a) Illustration of the preparation of the platform, by an origami folding. The detection antibodies are impregnated in cellulose, while capture antibodies are covalently immobilized in the detection pad. (b) The testing procedure, where the sliding tab is used to control the flow and the serial step execution of the assay. Reproduced from Ref. [30] (Chen C. A et al. Three-dimensional origami paper-based device for portable immunoassay applications. Lab on a Chip. 2019;19(4):598-607) with permission from The Royal Society of Chemistry

with porous silicon-based optical biosensors, where the reflectivity changes of the latter upon target binding are monitored in real time, with no need for target labeling. This was shown for DNA and protein targets with up to 1,000-fold enhancement in sensitivity [35, 36]. Nevertheless, application of an external field does require peripheral equipment, which both increases the cost and complicates the setup of the system.

\section{Magnetic-Assisted Platforms}

Magnetic nano- and microparticles are used in LoC devices for fluid manipulation. In many cases, the particles in the fluid are actuated by applying a magnetic field to induce the mixing (which is often limited in microfluidic devices due to laminar flow) [37-41]. Moreover, the particles can be also used as carriers and labels to facilitate both transfer and separation of biomolecules [37-41]. Magnetic particles are commonly controlled by electromagnets, coils, or permanent magnets - all of which induce an external magnetic field - and often form supramolecular structures 

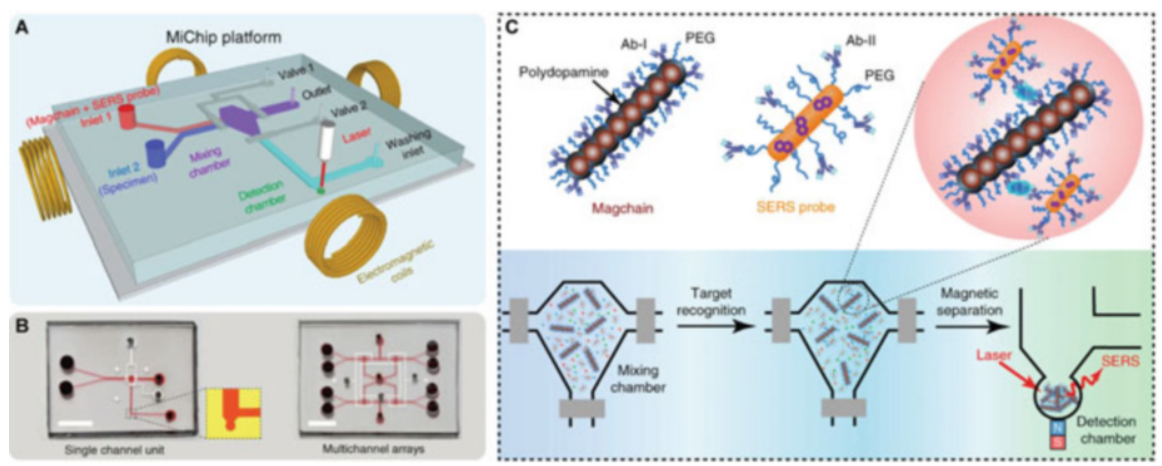

Fig. 4 Bio-conjugated magnetic nanochains on a microchip as rapid active liquid mixers and capture agents for bio-separation: (a) schematic illustration of the assay platform; (b) photographs of the platforms in a single- or multichannel format (scale bar: $0.5 \mathrm{~cm}$ ); and $(\mathbf{c})$ the detection assay: the sample, antibody-conjugated magnetic nanochains and surface-enhanced Raman spectroscopy (SERS)-encoded probes are mixed in the reaction chamber. Immune complexes are formed and isolated to the detection chambers, which are then subjected to Raman spectroscopic detection. Reprinted from Ref. [44] (Xiong Q. et al. Magnetic nanochain integrated microfluidic biochips. Nature Communications. 2018;9(1):1743). Copyright (C) 2018, Springer Nature

in the form of microcolumns due to dipole-dipole interactions [42, 43]. For example, bio-conjugated magnetic nanochains have been used on a microchip as stir bars to promote liquid mixing and as capture agents for bio-separation (see Fig. 4) [44]. A simple planar design of a microchip is realized based on flat channels on polydimethylsiloxane (PDMS)-on-glass, free of built-in components. The magnetic nanochains are biofunctionalized with target-specific antibodies, and surfaceenhanced Raman spectroscopy (SERS)-encoded nanoprobes are used as signaling probes for multiplexed Raman spectroscopic detection. A small amount $(\sim 1 \mu \mathrm{L})$ of sample fluid is mixed with both components, and the fluid flow and mixing are thereafter controlled via an external spinning magnetic field. Multiplexed detection of three cancer biomarkers in clinical serum and two bacterial species in saliva samples have been demonstrated in just 8 min [44].

Magnetic particles have been also used to automate processes in sandwich immunoassays, including the reaction and washing steps [45]. A sample is mixed with gold-coated iron oxide nanoparticles that have been functionalized with detection antibodies. The antibody-antigen reaction then forms immunocomplexes, which are electrochemically detected. The reaction and subsequent removal of unbound probes are controlled and accelerated by an external magnetic field. Thus, a simplified platform is obtained, without the need for fluid manipulation components and prestored washing buffer. Detection of a prostate-specific antigen in $10 \mu \mathrm{L}$ of human serum is demonstrated with a limit of detection of $0.085 \mathrm{ng} \mathrm{mL}^{-1}$ within $5 \mathrm{~min}$ [45]. A similar concept was used for developing a PoC multiplexed diagnostic test for differential detection of Ebola, Lassa, and malaria biomarkers in whole blood samples within $30 \mathrm{~min}$ [46]. Detection antibodies for the target antigens are conjugated to specific SERS nanotags and magnetic nanoparticles, which are stored dried 
in a test tube - providing a single-use and temperature stable platform that is ideal for field application. A whole blood sample $(45 \mu \mathrm{L})$ and a lysis buffer are added to rehydrate the dried reagents. After a mixing step, the magnetic microparticlesantigen-SERS nanotag complexes are separated with an external magnet, and an external laser is used for SERS signal monitoring [46]. Magnetic particles have been also used for signal amplification in lateral flow immunoassay strips for human chorionic gonadotropin detection $[47,48]$. For example, Pt-decorated magnetic coreshell nanoparticles, functionalized with detection antibodies, have been successfully deployed for this function [47]. These particles have both magnetic and enzyme-like properties, enabling target analyte magnetic enrichment and signal amplification by a peroxidase-like reaction mediated by the particles (see Fig. 5). The sensitivity is increased by two orders of magnitude when compared to a conventional lateral flow immunoassay [47].

In terms of nucleic acid analysis, magnetic particles are utilized for extraction, purification, amplification, and detection [49-51]. For nucleic acid amplification, isothermal methods are preferable for PoC testing, since they avoid the required

Step 1: Magnetic enrichment

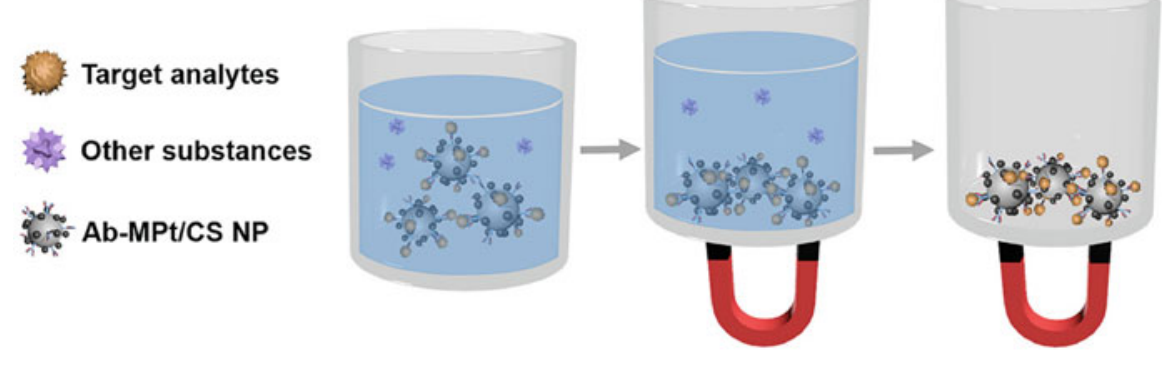

Step 2: Detection using LFIA

Step 3: Nanozyme-mediated signal amplification

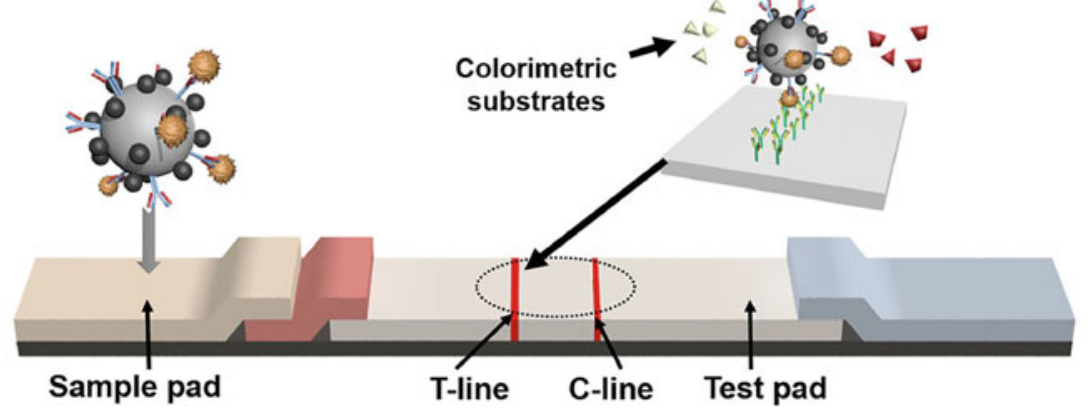

Fig. 5 Magnetic particles for signal amplification in lateral flow immunoassay (LFIA) for human chorionic gonadotropin detection. The particles have both magnetic and enzyme-like properties, enabling target analyte magnetic enrichment and signal amplification. Reprinted with permission from Ref. [47] (Kim M. S. et al. Pt-Decorated Magnetic Nanozymes for Facile and Sensitive Pointof-Care Bioassay. ACS Applied Materials \& Interfaces. 2017;9(40):35133-40). Copyright (2020) American Chemical Society 
thermal cycling in polymerase chain reaction [52-54]. One such method is so-called rolling circle amplification [55]: DNA or RNA target is annealed and ligated to a padlock probe, forming a circular template. The probe is highly sensitive to single base mutations, which results in high specificity [56]. Amplification reaction then proceeds via a phi29 polymerase, which creates a long single-stranded DNA concatemer containing repeated copies of the sequence complementary to the padlock probe [55]. Although this is a highly efficient isothermal method, the multiple steps in the assay and the different required reagents make the integration of this method onto a single-chip platform a challenging project. Magnetic particles can in turn facilitate the automation of the multistep assay [42, 57, 58]. For instance, a magnetic fluidized bed was recently integrated in a simple microfluidic chamber, generating a constant hydrodynamic recirculation in a continuous flow and thereby enabling efficient liquid perfusion and mixing [42]. The magnetic particles are functionalized with an oligonucleotide for the capture of the target DNA. A complete rolling circle amplification assay is performed on chip, with detection carried out in a low-cost polymer-based microarray module by fluorescence microscopy. The platform enables processing of large sample volumes, and a limit of detection of $1 \mathrm{pM}$ is obtained [42]. A similar concept is presented in a multichamber polymer-based microfluidic chip, which integrates DNA target capture, transport, and a rolling circle amplification assay, using magnetic microbeads [57]. The platform requires the manual loading of reagents, after which the assay runs automatically in a sequential chamber filling by capillary stop valves and phase guide structures. Opto-magnetic detection of a synthetic DNA target for type-B influenza virus is realized in $45 \mathrm{~min}$, with a limit of detection of $20 \mathrm{pM}$ [57].

\section{Centrifugal Microfluidic Platforms}

Multiplexed LoC detection can also be achieved by centrifugal microfluidics, which have been applied for detection of a wide range of analytes and have been thoroughly reviewed in the past [59-62]. The technology is based on a "Lab-on-a-CD" concept, wherein the complete fluidic network and the analysis steps are all embedded onto a single disc. The fluidic processing steps - including separation and reagent mixing - are then automated by implementing different spinning profiles. Integration of multiple assays in a single platform can thereby be achieved. The main advantage of these systems is their simple method of fluid manipulation, which is achieved by a rotary motor without the need for external pumps or a high-voltage power supply. The disc can also be synthesized from low-cost polymers, which facilitate both mass production and economical disposal. The Lab-on-a-CD technology has been successfully utilized for $\mathrm{PoC}$ diagnostics by several commercial companies. For example, Piccolo Xpress by Abaxis Inc., USA, [63] offers a variety of CD-based blood chemistry analyzers with up to 14 tests on a single disc. The platform requires only $0.1 \mathrm{~mL}$ of a blood sample, and results are obtained within $12 \mathrm{~min}$. Recently, the centrifugal microfluidic technology was also applied for a low 


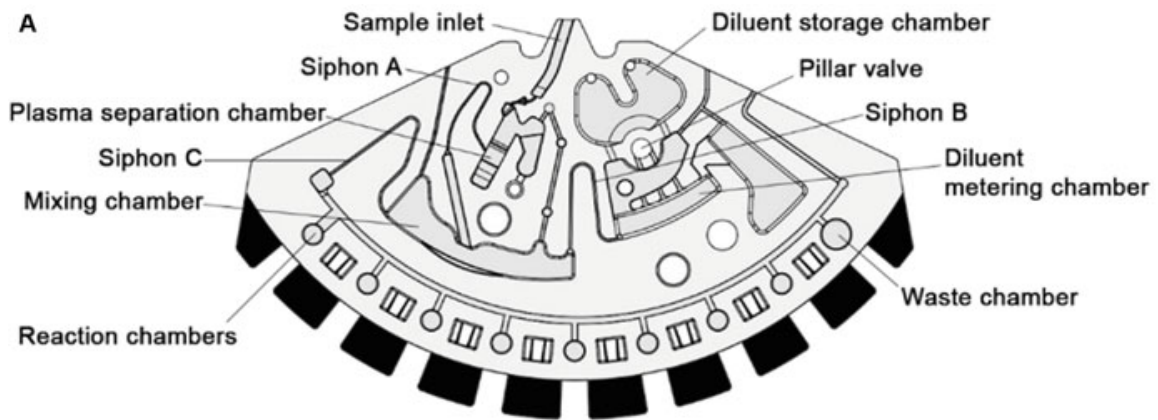

B

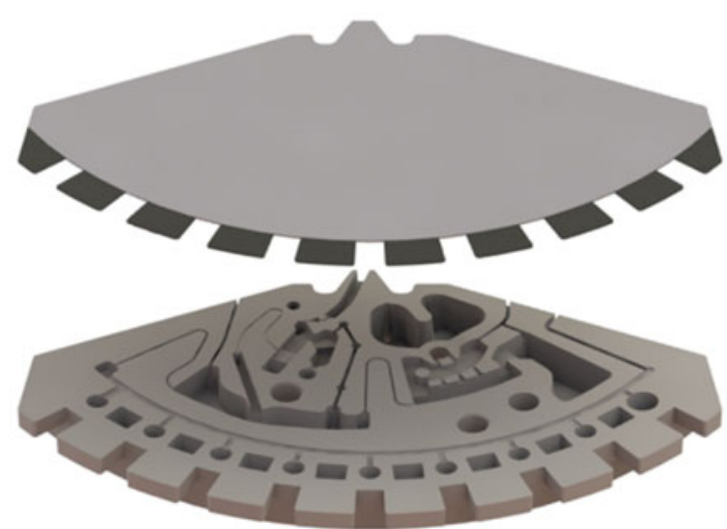

C

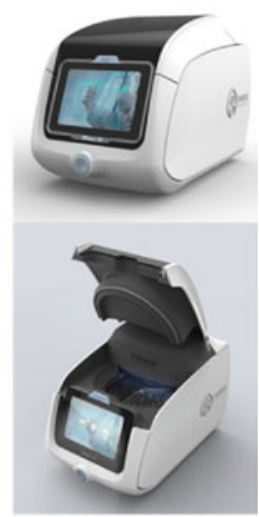

Fig. 6 (a) The design of the centrifugal microfluidic finger-prick blood biochemical analyzer; (b) exploded view of the chip, presenting an upper adhesive tape and bottom polycarbonate layer; (c) the portable biochemical analyzer. Reprinted with permission from Ref. [64] (Zhu Y. et al. Selfserved and fully automated biochemical detection of finger-prick blood at home using a portable microfluidic analyzer. Sensors and Actuators B: Chemical. 2020;303:127235). Copyright (C) 2019 Elsevier B.V

volume blood analysis, using only $12 \mu \mathrm{L}$ of blood from a finger prick, for automatic monitoring of blood glucose, total cholesterol, and triglycerides within $~ 15$ min (see Fig. 6a, b). Plasma separation, mixing, reaction, and detection are fully automated with a portable analyzer (Fig. 6c), which shows great potential for blood monitoring at home [64].

Centrifugal microfluidics is especially advantageous for nucleic acid detection, which requires lengthy and laborious sample preprocessing steps such as cell lysis, DNA purification, and amplification [65, 66]. Using this approach, all these steps can be integrated into a single disc and performed automatically and sequentially. For example, a centrifugal microfluidic device was integrated with a 3D-printed solution-loading cartridge for multiplex foodborne pathogen detection, as illustrated in Fig. 7a. The solution-loading cartridge prestores all required solutions for molecular diagnostics and connects with the reservoirs on the centrifugal device - minimizing 

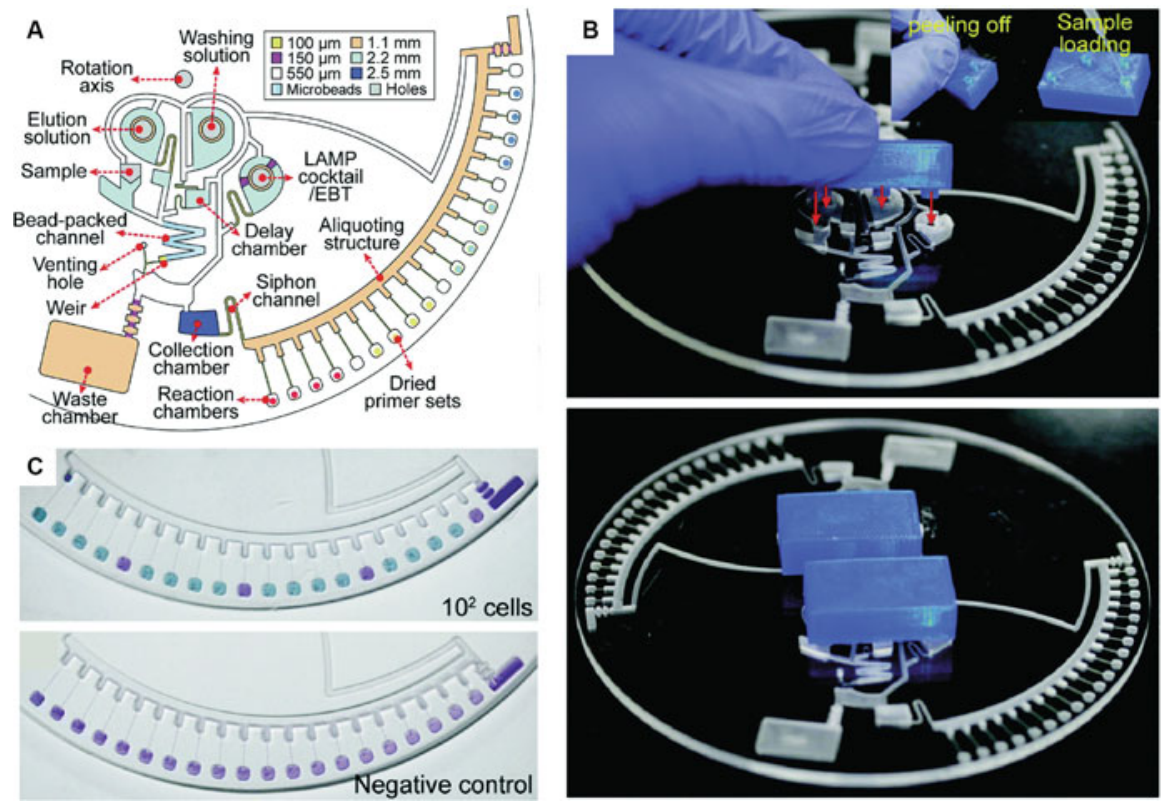

Fig. 7 (a) Design of the centrifugal microfluidics device for multiplex foodborne pathogen detection; (b) a real photograph of the microdevice with the solution loading and reagent storage cartridge; (c) multiplexed colorimetric detection of four different pathogens in milk sample in comparison to a negative control of pure milk. Reproduced from Ref. [67] (Oh S.J. and Seo T.S. Combination of a centrifugal microfluidic device with a solution-loading cartridge for fully automatic molecular diagnostics. Analyst. 2019;144(19):5766-74) with permission from The Royal Society of Chemistry

manual processing (see Fig. 7b). Sequential loading of the solutions to the device is achieved by controlling the rotational speed, and silica bead-assisted DNA extraction, isothermal DNA amplification, and colorimetric detection by Eriochrome Black $\mathrm{T}$ are then carried out. The platform enables detection of four kinds of foodborne pathogens in a real milk sample within 65 min and with a limit of detection of $10^{3}$ cells per mL (see Fig. 7c) [67]. Another technology which enables integration of DNA processing is double rotation axes centrifugal microfluidics, in which the disc can rotate around two rotation shafts - thus not limiting the fluid flow only radially outwards [68, 69]. This technology has allowed for a completely automated sample-to-result analysis of hepatitis B virus in whole blood [70]. The disc comprises all process chains for the virus DNA detection, including plasma separation from whole blood, lysis, DNA purification, and amplification. The double rotation axes centrifugal microfluidics allow for unconstrained and reversible fluid pumping, as well as an efficient spatial utilization of the disc. All reagents are prestored on the disc, and their introduction is controlled by melting ferrowax plugs in the channels with laser irradiation. The only manual step in the assay is 
the supply of $0.5 \mathrm{~mL}$ of a whole blood sample, while the time-to-result is $48 \mathrm{~min}$, with a limit of detection of $10^{2}$ copies per $\mathrm{mL}$ [70].

\section{Smartphone-Based Detection}

The high availability of smartphones worldwide and their sophisticated technological features (such as high quality cameras, connectivity, and computational power) have increasingly led to their integration into a wide range of analytical sensing systems [71-78]. Detection via smartphone is commonly based on various forms of optical measurements - including bright-field, colorimetric, luminescence, and/or fluorescence [71, 72]. The high resolution of the embedded complementary metal oxide semiconductor image sensor cameras enables high pixel density for optical monitoring, while the high computational power facilitates real-time image analysis [76]. Because smartphone-based PoC platforms have been extensively reviewed in the past few years [71-79], in this section we only briefly survey the main aspects of smartphone-based detection with a few examples from recent years.

Bright-field-based detection is the simplest method, where a sample is illuminated from below with white light and then the transmitted light is measured [72]. Imaging of living cells or large biomolecules can be achieved in this way [80, 81]. Colorimetric-based detection is also relatively simple, requiring only illumination and image processing. This has been commonly used in connection with paper-based assays to achieve quantitative results. For example, a custom-built smartphone application was used to quantitate a PoC lateral flow assay for detection of Ebola virus-specific antibodies in clinical human serum samples. This low-cost platform requires only the test strip and a smartphone, and results are obtained within 15 min [82]. Smartphone colorimetric detection of lactate dehydrogenase as a biomarker for cellular damage for early diagnosis of serious illness in neonates was also recently shown, as illustrated in Fig. 8 [83]. The PoC platform consists of a plastic cartridge holding disposable filter papers for whole blood filtration, plasma separation, and colorimetric reaction. The cartridge is mounted in a box (Fig. 8b), which also holds the smartphone at a fixed distance for automatic imaging. A dedicated application is used for analyzing the RGB values of the acquired images, and comparable results to standard laboratory analysis are obtained in only $4 \mathrm{~min}$ [83]. Colorimetric-based detection using a multichannel smartphone spectrometer as an optical biosensor was recently used to detect protein content and a cancer biomarker within human serum [84]. Images captured by the phone camera were converted to transmission and absorbance spectra in the visible light range with high resolution, and the performance of the setup was comparable to benchtop instruments.

To increase the sensitivity of the assay, fluorescence-based detection is also frequently employed. For such systems, an optomechanical modulus containing excitation and/or emission filter and laser diodes for excitation are required [76]. For example, a compact multimodal microscope was integrated on a smartphone for targeted DNA sequencing and in situ point mutation analysis in 


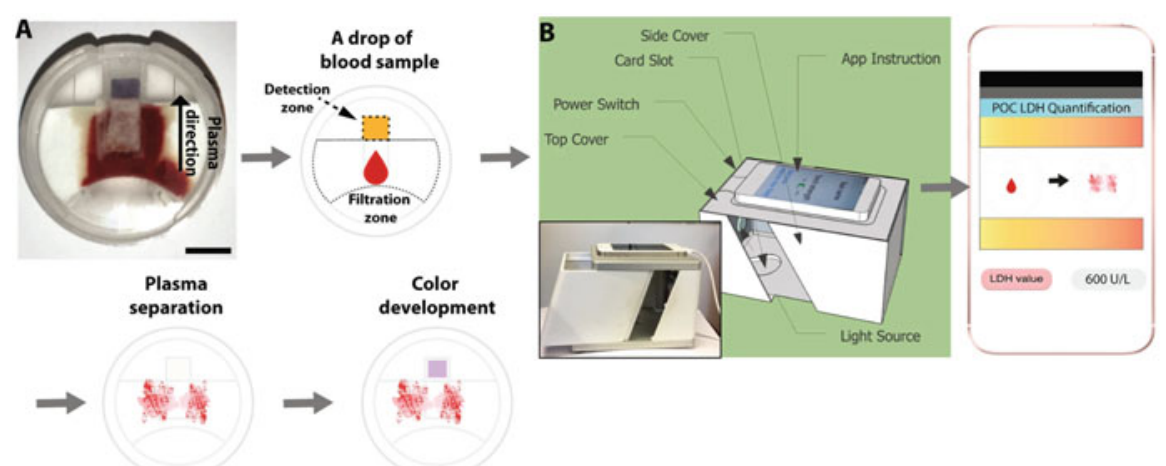

Fig. 8 (a) Schematics of a PoC lateral flow assay system for analysis of lactate dehydrogenase (LDH) in whole blood, consisting of a plastic cartridge holding filter papers. Scale bar: $0.4 \mathrm{~cm}$. (b) The cartridge is placed on a designated slot inside a box; the latter keeps a fix distance between the phone camera and the cartridge for the imaging while ensuring similar light conditions between different batches. An app is used to guide the user in the assay and analyze the results. Reprinted from Ref. [83] (Halvorsen C.P., et al. A rapid smartphone-based lactate dehydrogenase test for neonatal diagnostics at the point-of-care. Scientific Reports. 2019;9(1):9301). Copyright (C) 2019, Springer Nature

tumor samples [85]. A 3D-printed lightweight optomechanical modulus is integrated on the smartphone and contains two laser diodes for multicolor fluorescence imaging, as well as a white light-emitting diode (LED) for bright-field transmission imaging. DNA sequencing and point mutation analysis are achieved via rolling circle amplification, and the results are comparable to regular benchtop microscopes. Such technology is applicable for genotyping cancer patient biopsies directly in the pathologist office at PoC. Similar concept was shown for multiplexed detection of Zika, chikungunya, and dengue viruses (belonging to the Flaviviridae family) directly in human blood, saliva, and urine samples (see Fig. 9) [86]. This platform is comprised of three components: a heating module, a reaction module, and an optical-detection and image analysis module. The latter contains multicolored LED coupled with a multi-pass band filter for fluorescence measurement. The entire platform is fitted with a smartphone, and the camera is utilized for the imaging. A dedicated application is used for fluorescence signal analysis by a novel algorithm, improving the discrimination between positive and negative signals by fivefold, compared to a naked eye. Target virus RNAs are detected by reverse-transcription loop-mediated isothermal amplification coupled with quenching of unincorporated amplification signal reporters. Recently, microfluidic-based immunoassay based on a smartphone fluorescence detection was used to conduct troponin I analysis in human serum in clinically relevant concentrations within 12 min [87]. Although fluorescence-based detection improves the sensitivity of the assay, it also requires the addition of complex and costly optical components to the system. Time-gated photoluminescence-based detection may offer one economical alternative. This concept is demonstrated for human chorionic gonadotropin quantification in a lateral 

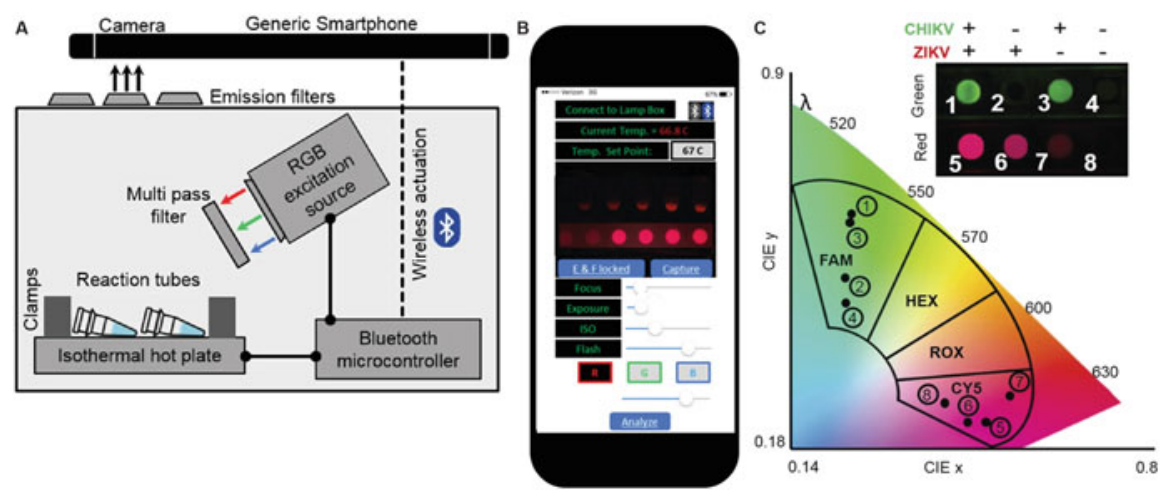

Fig. 9 (a) Schematic illustration of the smartphone-based fluorescence detection of Zika, chikungunya, and dengue virus's RNA platform, based on reverse-transcription loop-mediated isothermal amplification assay. The system comprises isothermal heating unit with reaction tubes, LED excitation source and Bluetooth microcontroller. (b) An app is used to wirelessly actuate the isothermal heater and excitation source. The smartphone camera with an emission filter captures the images, analyzed subsequently by the app. (c) Duplex detection of Zika and chikungunya viruses. The images are mapped over predefined fluorophore emission islands to distinguish between different viral targets. Adapted with changes from Ref. [86]. (Priye A. et al. A smartphone-based diagnostic platform for rapid detection of Zika, chikungunya, and dengue viruses. Scientific Reports. 2017;7(1):44778). Copyright (C) 2017, Springer Nature

flow assay with a persistent luminescent phosphor reporter [88]. A smartphone's flash is used to excite the nanophosphors, which are then imaged using the smartphone camera. A 10- to 100-fold enhancement in sensitivity is achieved compared to commercial lateral flow assays - without the need for any additional complex optical components.

\section{Conclusions and Outlook}

Significant research efforts have already been directed towards the development of simple and low-cost devices for LoC-based medical diagnostics at PoC. Nevertheless, commercialization of such technologies remains limited, and the following aspects must be considered:

- Real PoC application (in terms of sample in - results out) requires the integration and automation of all assay steps - yet most assays still require extensive user intervention, mainly in terms of sample preparation and/or reagent addition. For certain applications, this bottleneck can perhaps be solved by the integration of reagent moduli and, where possible, simple reagent storage on the chip.

- The need to improve the sensitivity, selectivity, and stability of sensing moduli is increasingly leading researchers to explore robust recognition elements - such as 
aptamers, antibody fragments, and molecularly imprinted polymers. Various nanomaterials are also being incorporated for signal amplification and to improve the total assay performance.

- Scalability is an essential requirement for commercialization, and it continues to pose profound challenges for complex microfluidic structures. As a result, scalability considerations must direct the materials, design, and fabrication methods that are employed for such devices. For example, PDMS (which is commonly used for microfluidic fabrication in the academy) is not suitable for mass production, since it is fabricated mostly via soft lithography techniques. As a result, gold-standard paper-based assays continue to rule the field of PoC diagnostics by dint of the fact that they can be mass-produced at a very low-cost. Advancements in 3D-printing technology will likely begin to close that gap in the near future, at least with respect to plastic-based microfluidics.

- Smartphone technology has expanded the analytical power and increased the accessibility of many platforms. But hygiene considerations - including both contamination and disposal issues - must be carefully considered if smartphones will be deployed.

- Because multiplexing for the simultaneous detection of several biomarkers is extremely valuable in the context of medical diagnostics, the authors anticipate that research efforts in this direction will continue to increase exponentially.

- Clinical validation of all platforms is required. Many of the published works utilize human biological samples spiked with the analyte; although this is sufficient for a proof-of-concept, real clinical samples from different patients should always be tested in order to validate a platform's design integrity.

- Finally, the social impact of this emerging technology - as well as corresponding regulatory policies and concerns - should be considered when designing an assay, in order to facilitate (or at least preserve) its commercialization potential.

\section{References}

1. Sorger PK (2008) Microfluidics closes in on point-of-care assays. Nat Biotechnol 26 (12):1345-1346

2. Sanjay ST, Fu G, Dou M, Xu F, Liu R, Qi H et al (2015) Biomarker detection for disease diagnosis using cost-effective microfluidic platforms. Analyst 140(21):7062-7081

3. Lee J, Lee S-H (2013) Lab on a chip for in situ diagnosis: from blood to point of care. Biomed Eng Lett 3(2):59-66

4. Gubala V, Harris LF, Ricco AJ, Tan MX, Williams DE (2012) Point of care diagnostics: status and future. Anal Chem 84(2):487-515

5. Jung W, Han J, Choi J-W, Ahn CH (2015) Point-of-care testing (POCT) diagnostic systems using microfluidic lab-on-a-chip technologies. Microelectron Eng 132:46-57

6. Kost GJ (1995) Guidelines for point-of-care testing. Improving patient outcomes. Am J Clin Pathol 104(4 Suppl 1):S111-S127

7. Luppa PB, Müller C, Schlichtiger A, Schlebusch H (2011) Point-of-care testing (POCT): current techniques and future perspectives. TrAC Trends Anal Chem 30(6):887-898

8. Whitesides GM (2006) The origins and the future of microfluidics. Nature 442(7101):368-373 
9. Zhang Z, Nagrath S (2013) Microfluidics and cancer: are we there yet? Biomed Microdevices 15(4):595-609

10. Volpatti LR, Yetisen AK (2014) Commercialization of microfluidic devices. Trends Biotechnol 32(7):347-350

11. Haeberle S, Zengerle R (2007) Microfluidic platforms for lab-on-a-chip applications. Lab Chip 7(9):1094-1110

12. Schumacher S, Nestler J, Otto T, Wegener M, Ehrentreich-Förster E, Michel D et al (2012) Highly-integrated lab-on-chip system for point-of-care multiparameter analysis. Lab Chip 12 (3):464-473

13. Eicher D, Merten CA (2011) Microfluidic devices for diagnostic applications. Expert Rev Mol Diagn 11(5):505-519

14. Koczula Katarzyna M, Gallotta A (2016) Lateral flow assays. Essays Biochem 60(1):111-120

15. Anfossi L, Di Nardo F, Cavalera S, Giovannoli C, Baggiani C (2018) Multiplex lateral flow immunoassay: an overview of strategies towards high-throughput point-of-need testing. Biosensors 9(1):2

16. Hemmig E, Temiz Y, Gökçe O, Lovchik RD, Delamarche E (2020) Transposing lateral flow immunoassays to capillary-driven microfluidics using self-coalescence modules and capillaryassembled receptor carriers. Anal Chem 92(1):940-946

17. Carrell C, Kava A, Nguyen M, Menger R, Munshi Z, Call Z et al (2019) Beyond the lateral flow assay: a review of paper-based microfluidics. Microelectron Eng 206:45-54

18. Yetisen AK, Akram MS, Lowe CR (2013) Paper-based microfluidic point-of-care diagnostic devices. Lab Chip 13(12):2210-2251

19. Gong MM, Sinton D (2017) Turning the page: advancing paper-based microfluidics for broad diagnostic application. Chem Rev 117(12):8447-8480

20. Channon RB, Nguyen MP, Scorzelli AG, Henry EM, Volckens J, Dandy DS et al (2018) Rapid flow in multilayer microfluidic paper-based analytical devices. Lab Chip 18(5):793-802

21. Magro L, Escadafal C, Garneret P, Jacquelin B, Kwasiborski A, Manuguerra J-C et al (2017) Paper microfluidics for nucleic acid amplification testing (NAAT) of infectious diseases. Lab Chip 17(14):2347-2371

22. Tian T, Bi Y, Xu X, Zhu Z, Yang C (2018) Integrated paper-based microfluidic devices for point-of-care testing. Anal Methods 10(29):3567-3581

23. Gervais L, de Rooij N, Delamarche E (2011) Microfluidic chips for point-of-care immunodiagnostics. Adv Mater 23(24):H151-HH76

24. Song Y, Lin B, Tian T, Xu X, Wang W, Ruan Q et al (2019) Recent progress in microfluidicsbased biosensing. Anal Chem 91(1):388-404

25. Apple FS, Christenson RH, Valdes Jr R, Andriak AJ, Berg A, Duh S-H et al (2020) Simultaneous rapid measurement of whole blood myoglobin, creatine kinase $\mathrm{MB}$, and cardiac troponin I by the triage cardiac panel for detection of myocardial infarction. Clin Chem 45(2):199-205

26. Clark TJ, McPherson PH, Buechler KF (2002) The triage cardiac panel: cardiac markers for the triage system. Point Care 1(1):42-46

27. Wang J, Ahmad H, Ma C, Shi Q, Vermesh O, Vermesh U et al (2010) A self-powered, one-step chip for rapid, quantitative and multiplexed detection of proteins from pinpricks of whole blood. Lab Chip 10(22):3157-3162

28. Gökçe O, Castonguay S, Temiz Y, Gervais T, Delamarche E (2019) Self-coalescing flows in microfluidics for pulse-shaped delivery of reagents. Nature 574(7777):228-232

29. Liu H, Crooks RM (2011) Three-dimensional paper microfluidic devices assembled using the principles of origami. J Am Chem Soc 133(44):17564-17566

30. Chen C-A, Yeh W-S, Tsai T-T, Li Y-D, Chen C-F (2019) Three-dimensional origami paperbased device for portable immunoassay applications. Lab Chip 19(4):598-607

31. Liu L, Yang D, Liu G (2019) Signal amplification strategies for paper-based analytical devices. Biosens Bioelectron 136:60-75

32. Bishop JD, Hsieh HV, Gasperino DJ, Weigl BH (2019) Sensitivity enhancement in lateral flow assays: a systems perspective. Lab Chip 19(15):2486-2499 
33. Moghadam BY, Connelly KT, Posner JD (2014) Isotachophoretic preconcenetration on paperbased microfluidic devices. Anal Chem 86(12):5829-5837

34. Guo S, Schlecht W, Li L, Dong W-J (2019) Paper-based cascade cationic isotachophoresis: multiplex detection of cardiac markers. Talanta 205:120112

35. Arshavsky-Graham S, Massad-Ivanir N, Paratore F, Scheper T, Bercovici M, Segal E (2017) On chip protein pre-concentration for enhancing the sensitivity of porous silicon biosensors. ACS Sensors 2(12):1767-1773

36. Vilensky R, Bercovici M, Segal E (2015) Oxidized porous silicon nanostructures enabling electrokinetic transport for enhanced DNA detection. Adv Funct Mater 25(43):6725-6732

37. Moerland CP, van Ijzendoorn LJ, Prins MWJ (2019) Rotating magnetic particles for lab-onchip applications - a comprehensive review. Lab Chip 19(6):919-933

38. Chircov C, Grumezescu AM, Holban AM (2019) Magnetic particles for advanced molecular diagnosis. Materials 12(13):2158

39. van Reenen A, de Jong AM, den Toonder JMJ, Prins MWJ (2014) Integrated lab-on-chip biosensing systems based on magnetic particle actuation - a comprehensive review. Lab Chip 14(12):1966-1986

40. Ríos Á, Zougagh M (2016) Recent advances in magnetic nanomaterials for improving analytical processes. TrAC Trends Anal Chem 84:72-83

41. Masud MK, Na J, Younus M, Hossain MSA, Bando Y, Shiddiky MJA et al (2019) Superparamagnetic nanoarchitectures for disease-specific biomarker detection. Chem Soc Rev 48(24):5717-5751

42. Hernández-Neuta I, Pereiro I, Ahlford A, Ferraro D, Zhang Q, Viovy J-L et al (2018) Microfluidic magnetic fluidized bed for DNA analysis in continuous flow mode. Biosens Bioelectron 102:531-539

43. Lacharme F, Vandevyver C, Gijs MAM (2008) Full on-chip nanoliter immunoassay by geometrical magnetic trapping of nanoparticle chains. Anal Chem 80(8):2905-2910

44. Xiong Q, Lim CY, Ren J, Zhou J, Pu K, Chan-Park MB et al (2018) Magnetic nanochain integrated microfluidic biochips. Nat Commun 9(1):1743

45. Hwang H, Choi E, Han S, Lee Y, Choi T, Kim M et al (2019) MESIA: magnetic force-assisted electrochemical sandwich immunoassays for quantification of prostate-specific antigen in human serum. Anal Chim Acta 1061:92-100

46. Sebba D, Lastovich AG, Kuroda M, Fallows E, Johnson J, Ahouidi A et al (2018) A point-ofcare diagnostic for differentiating Ebola from endemic febrile diseases. Sci Trans Med 10(471): eaat0944

47. Kim MS, Kweon SH, Cho S, An SSA, Kim MI, Doh J et al (2017) Pt-decorated magnetic nanozymes for facile and sensitive point-of-care bioassay. ACS Appl Mater Interfaces 9 (40):35133-35140

48. Jacinto MJ, Trabuco JRC, Vu BV, Garvey G, Khodadady M, Azevedo AM et al (2018) Enhancement of lateral flow assay performance by electromagnetic relocation of reporter particles. PLoS One 13:e0186782

49. Tamanaha CR, Mulvaney SP, Rife JC, Whitman LJ (2008) Magnetic labeling, detection, and system integration. Biosens Bioelectron 24(1):1-13

50. Kojima T, Takei Y, Ohtsuka M, Kawarasaki Y, Yamane T, Nakano H (2005) PCR amplification from single DNA molecules on magnetic beads in emulsion: application for highthroughput screening of transcription factor targets. Nucl Acids Res 33(17):e150-e

51. Berensmeier S (2006) Magnetic particles for the separation and purification of nucleic acids. Appl Microbiol Biotechnol 73(3):495-504

52. Zhao Y, Chen F, Li Q, Wang L, Fan C (2015) Isothermal amplification of nucleic acids. Chem Rev 115(22):12491-12545

53. Duan R, Lou X, Xia F (2016) The development of nanostructure assisted isothermal amplification in biosensors. Chem Soc Rev 45(6):1738-1749

54. Deng H, Gao Z (2015) Bioanalytical applications of isothermal nucleic acid amplification techniques. Anal Chim Acta 853:30-45 
55. Ali MM, Li F, Zhang Z, Zhang K, Kang D-K, Ankrum JA et al (2014) Rolling circle amplification: a versatile tool for chemical biology, materials science and medicine. Chem Soc Rev 43(10):3324-3341

56. Nilsson M, Gullberg M, Dahl F, Szuhai K, Raap AK (2002) Real-time monitoring of rollingcircle amplification using a modified molecular beacon design. Nucl Acids Res 30(14):e66-e

57. Garbarino F, Minero GAS, Rizzi G, Fock J, Hansen MF (2019) Integration of rolling circle amplification and optomagnetic detection on a polymer chip. Biosens Bioelectron 142:111485

58. Minero GAS, Cangiano V, Garbarino F, Fock J, Hansen MF (2019) Integration of microbead DNA handling with optomagnetic detection in rolling circle amplification assays. Microchim Acta 186(8):528

59. Gorkin R, Park J, Siegrist J, Amasia M, Lee BS, Park J-M et al (2010) Centrifugal microfluidics for biomedical applications. Lab Chip 10(14):1758-1773

60. Tang M, Wang G, Kong S-K, Ho H-P (2016) A review of biomedical centrifugal microfluidic platforms. Micromachines 7(2):26

61. Burger R, Amato L, Boisen A (2016) Detection methods for centrifugal microfluidic platforms. Biosens Bioelectron 76:54-67

62. Strohmeier O, Keller M, Schwemmer F, Zehnle S, Mark D, von Stetten F et al (2015) Centrifugal microfluidic platforms: advanced unit operations and applications. Chem Soc Rev 44(17):6187-6229

63. Piccolo Xpress: Abaxis Inc (2019) https://www.abaxis.com/medical/piccolo-xpress

64. Zhu Y, Meng X, Chen Y, Li J, Shao H, Lu Y et al (2020) Self-served and fully automated biochemical detection of finger-prick blood at home using a portable microfluidic analyzer. Sensors Actuators B Chem 303:127235

65. Amasia M, Cozzens M, Madou MJ (2012) Centrifugal microfluidic platform for rapid PCR amplification using integrated thermoelectric heating and ice-valving. Sensors Actuators B Chem 161(1):1191-1197

66. Czilwik G, Messinger T, Strohmeier O, Wadle S, von Stetten F, Paust N et al (2015) Rapid and fully automated bacterial pathogen detection on a centrifugal-microfluidic LabDisk using highly sensitive nested PCR with integrated sample preparation. Lab Chip 15(18):3749-3759

67. Oh SJ, Seo TS (2019) Combination of a centrifugal microfluidic device with a solution-loading cartridge for fully automatic molecular diagnostics. Analyst 144(19):5766-5774

68. Cao X, de Mello AJ, Elvira KS (2016) Enhanced versatility of fluid control in centrifugal microfluidic platforms using two degrees of freedom. Lab Chip 16(7):1197-1205

69. Zhu Y, Chen Y, Meng X, Wang J, Lu Y, Xu Y et al (2017) Comprehensive study of the flow control strategy in a wirelessly charged centrifugal microfluidic platform with two rotation axes. Anal Chem 89(17):9315-9321

70. Li L, Miao B, Li Z, Sun Z, Peng N (2019) Sample-to-answer hepatitis B virus DNA detection from whole blood on a centrifugal microfluidic platform with double rotation axes. ACS Sensors 4(10):2738-2745

71. Kanchi S, Sabela MI, Mdluli PS, Inamuddin, Bisetty K (2018) Smartphone based bioanalytical and diagnosis applications: a review. Biosens Bioelectron 102:136-149

72. Liu J, Geng Z, Fan Z, Liu J, Chen H (2019) Point-of-care testing based on smartphone: the current state-of-the-art (2017-2018). Biosens Bioelectron 132:17-37

73. Vashist SK, Mudanyali O, Schneider EM, Zengerle R, Ozcan A (2014) Cellphone-based devices for bioanalytical sciences. Anal Bioanal Chem 406(14):3263-3277

74. Contreras-Naranjo JC, Wei Q, Ozcan A (2016) Mobile phone-based microscopy, sensing, and diagnostics. IEEE J Sel Top Quantum Electron 22(3):1-14

75. Dutta S (2019) Point of care sensing and biosensing using ambient light sensor of smartphone: critical review. TrAC Trends Anal Chem 110:393-400

76. Xu D, Huang X, Guo J, Ma X (2018) Automatic smartphone-based microfluidic biosensor system at the point of care. Biosens Bioelectron 110:78-88 
77. Vashist SK, Luong JHT (2019) Smartphone-based point-of-care technologies for mobile healthcare. In: Vashist SK, Luong JHT (eds) Point-of-care technologies enabling nextgeneration healthcare monitoring and management. Springer, Cham, pp 27-79

78. Hernández-Neuta I, Neumann F, Brightmeyer J, Ba Tis T, Madaboosi N, Wei Q et al (2019) Smartphone-based clinical diagnostics: towards democratization of evidence-based health care. J Intern Med 285(1):19-39

79. Arumugam S, Colburn DAM, Sia SK Biosensors for personal mobile health: a system architecture perspective. Adv Mater Technol:1900720

80. Kanakasabapathy MK, Pandya HJ, Draz MS, Chug MK, Sadasivam M, Kumar S et al (2017) Rapid, label-free CD4 testing using a smartphone compatible device. Lab Chip 17 (17):2910-2919

81. Kanakasabapathy MK, Sadasivam M, Singh A, Preston C, Thirumalaraju P, Venkataraman M et al (2017) An automated smartphone-based diagnostic assay for point-of-care semen analysis. Sci Trans Med 9(382):eaai7863

82. Brangel P, Sobarzo A, Parolo C, Miller BS, Howes PD, Gelkop S et al (2018) A serological point-of-care test for the detection of IgG antibodies against Ebola virus in human survivors. ACS Nano 12(1):63-73

83. Halvorsen CP, Olson L, Araújo AC, Karlsson M, Nguyễn TT, Khu DTK et al (2019) A rapid smartphone-based lactate dehydrogenase test for neonatal diagnostics at the point of care. Sci Rep 9(1):9301

84. Wang L-J, Chang Y-C, Sun R, Li L (2017) A multichannel smartphone optical biosensor for high-throughput point-of-care diagnostics. Biosens Bioelectron 87:686-692

85. Kühnemund M, Wei Q, Darai E, Wang Y, Hernández-Neuta I, Yang Z et al (2017) Targeted DNA sequencing and in situ mutation analysis using mobile phone microscopy. Nat Commun 8 (1):13913

86. Priye A, Bird SW, Light YK, Ball CS, Negrete OA, Meagher RJ (2017) A smartphone-based diagnostic platform for rapid detection of Zika, chikungunya, and dengue viruses. Sci Rep 7 (1):44778

87. Liang C, Liu Y, Niu A, Liu C, Li J, Ning D (2019) Smartphone-app based point-of-care testing for myocardial infarction biomarker cTnI using an autonomous capillary microfluidic chip with self-aligned on-chip focusing (SOF) lenses. Lab Chip 19(10):1797-1807

88. Paterson AS, Raja B, Mandadi V, Townsend B, Lee M, Buell A et al (2017) A low-cost smartphone-based platform for highly sensitive point-of-care testing with persistent luminescent phosphors. Lab Chip 17(6):1051-1059 